Pathologe 2008 $\cdot 29: 172-174$

DOI 10.1007/s00292-008-0994-2

Online publiziert: 15. März 2008

๑) Springer Medizin Verlag 2008

\section{G. Jundt}

Institut für Pathologie, DÖSAK-Zentralregister am Knochentumor-

Referenzzentrum, Universitätsspital Basel, Schweiz

\title{
Odontogene Tumoren und Zysten im Kiefer
}

\section{Schwierige Differenzialdiagnose}

Während Kieferzysten relativ häufig im diagnostischen Eingang vorkommen, sind odontogene Tumoren ausgesprochene Raritäten. Beide Läsionen können jedoch eine Reihe diagnostischer Probleme bieten, die dadurch noch erhöht werden, dass der Pathologe nur sehr selten Angaben über den exakten Entnahmeort und dessen Beziehung zu Zähnen oder die Panoramaschichtaufnahme als topographische $\mathrm{Zu}$ satzinformation erhält. Außerdem sind zahlreiche Läsionen so selten, dass außerhalb eines spezialisierten oralpathologischen Einganges kaum die nötige Erfahrung mit diesen Läsionen zu gewinnen ist. Die diagnostische "Etikettierung" hat aber therapeutische Konsequenzen.

Diese oft unbefriedigende Situation hat 1972 Kieferchirurgen aus Deutschland, Österreich und der Schweiz veranlasst, sich im deutsch-österreichisch-schweizerischen Arbeitskreis für Tumoren im Kiefer-Gesichtsbereich (DÖSAK) zusammenzuschließen und die Gründung eines Referenzregisters $z u$ initiieren, das am Institut für Pathologie des Universitätsspitals Basel beheimatet und in das schweizerische KnochentumorReferenzzentrum eingegliedert ist. Aus den Einsendungen an das Register lässt sich ein sehr guter Überblick über die Probleme gewinnen, die Kliniker und Pathologen mit der Diagnostik dieser Läsionen haben.

Im Vordergrund steht dabei die Abgrenzung odontogener Zysten von Formen des Ameloblastoms, die Abgrenzung odontogener Tumoren untereinander sowie die Unterscheidung normaler Strukturen, wie Fragmente eines Zahnfollikels oder der Zahnpapille, von odontogenen Tumoren.
Ein großer Teil dieser Fragen kann nur in enger Zusammenarbeit mit den Klinikern gelöst werden. Informationen über die Lagebeziehung einer Läsion zu einem Zahn, ihre Lokalisation im Kiefer und ihr Verhalten gegenüber der Umgebung, die sich aus dem Röntgenbild erschließen lässt, sind wichtigere Bausteine der Diagnose als immunhistochemische Zusatzuntersuchungen, die für die Differenzialdiagnose odontogener Tumoren und der Kieferzysten wegen ihrer Variationsbreite in der Regel keine verlässliche differenzialdiagnostische Hilfe bieten, weshalb auf sie in den folgenden Artikeln auch nur punktuell eingegangen wird. Die Stellung einer eindeutigen Diagnose ist aber die Basis für eine adäquate Therapie, die gerade bei odontogenen Tumoren fast immer eine operative Maßnahme beinhaltet. Diese reicht von einer einfachen Kürettage bis hin zur kompletten Kontinuitätsresektion mit entsprechendem Sicherheitsabstand und evtl. sogar einer Lymphknotenausräumung.

\section{Zahnentwicklung}

Odontogene Tumoren und Kieferzysten zeichnen sich durch eine große morphologische Vielfalt aus. Gleichzeitig gehören zumindest die odontogenen Tumoren, die sich vom Zahnkeim und vom Zahnhalteapparat ableiten lassen, $z u$ den seltensten $\mathrm{Tu}$ moren überhaupt. Dies ist erstaunlich, da die Zähne durch einen komplizierten doppelten Induktionsprozess gebildet werden, der interaktiv durch das Zusammenspiel von odontogenem Epithel und Ektomesenchym abläuft und mit der Aktivierung zahlreicher Transkriptionsfaktoren verbunden ist, die sich wechselseitig beeinflussen.
Dennoch scheint die Zahnentwicklung genetisch so fest fixiert zu sein, dass es kaum zu Störungen kommt, was sich an der geringen Zahl neu auftretender odontogener Tumoren ablesen lässt.

An der Bildung des Zahnes sind ektodermales odontogenes Epithel sowie Mesenchymanteile neuroektodermaler Herkunft, das Ektomesenchym, beteiligt, das neben Spindelzellen eine charakteristische gelatinöse Grundsubstanz besitzt [4].

Aus dem Epithel der Mundhöhle entwickelt sich durch Wachstum in das darunter liegende Ektomesenchym die Zahnleiste, die ihrerseits einen induktiven Effekt auf das Ektomesenchym ausübt, das sich verdichtet und die spätere Papille bildet. Die Spitze der Zahnleiste vergrößert sich gleichzeitig und bildet so das spätere Schmelzorgan, das eine Auflockerung des Zusammenhaltes seiner zentralen Epithelien zeigt, die als sternförmiges Retikulum bezeichnet wird. Durch die knotig konfigurierte ektomesenchymale Papillenanlage wird das sich entwickelnde Schmelzorgan kappenförmig eingedellt. Die äußeren und inneren kompakt angeordneten Epithelien, die das aufgelockerte sternförmige Retikulum des Schmelzorgans begrenzen, werden als inneres Schmelzepithel und äußeres Schmelzepithel bezeichnet. An der Basis der Papille gehen sie ineinander über und bilden dabei eine Art Schlaufe. Aus dieser entwickelt sich später die nach Hertwig benannte Wurzelscheide, die die Differenzierung von Odontoblasten induziert.

Das Schmelzorgan und das kondensierte, die spätere Papille bildende Ektomesenchym werden von kollagenbildenden Fibroblasten umgeben, die den Zahnfollikel bzw. das Zahnsäckchen bilden. Die Zellen 
der Papille, die der Hertwig-Wurzelscheide anliegen, differenzieren sich zu ektomesenchymalen Odontoblasten und bilden Dentin. Das hat andererseits eine induktive Wirkung auf das angrenzende Epithel, aus dem sich die Ameloblasten als hochprismatische Zellen formen und Schmelz auf das darunter liegende Dentin ablagern. Mit zunehmendem Wachstum des Zahns werden Epithelien von der Hertwig-Wurzelscheide abgelöst und bleiben im sich zwischen Zahn und Knochen bildenden Periodontalspalt als Malassez-Epithelnester liegen. Ektomesenchymale Zellen, die in Höhe der ehemaligen "Schlaufe" der HertwigWurzelscheide liegen, differenzieren sich $z u$ zementbildenden Zementoblasten. Schließlich löst sich auch der Zusammenhalt der Zahnleiste, und Epithelreste bleiben in einzelnen Verbänden zurück, den nach Serres benannten Epithelperlen.

Von diesen Strukturen (Zahnleiste, Serres-Epithelien, Schmelzorgan mit sternförmigem Retikulum, Zahnpapille, Zahnfollikel, Malassez-Zellreste, Ameloblasten, Odontoblasten und Zementoblasten) lassen sich die Tumoren und Zysten des zahnbildenden Gewebes ableiten (• Abb. 1).

\section{Klassifikation}

Die WHO-Klassifikation der odontogenen Tumoren von 2005 beruht im Wesentlichen auf den gleichen Prinzipien, die schon 1992 in der 2. Auflage angewendet wurden [1,3]. Neben der Unterscheidung zwischen (wesentlich häufigeren) gutartigen und (extrem seltenen) bösartigen Tumoren spielt bei der Klassifikation die morphologische Ähnlichkeit der Tumoren zur normalen Zahnentwicklung die wichtigste Rolle. Je nachdem, ob odontogenes Epithel, odontogenes Ektomesenchym oder eine Kombination von beiden die Tumorkomponenten bilden, können 3 verschiedene Gruppen unterschieden werden. Die Hartsubstanzbildung (Schmelz, Dentin) wird nur bei der weiteren Subklassifikation der einzelnen Gruppen berücksichtigt. Man kann deshalb zwischen Tumoren unterscheiden,

- die nur aus odontogenem Epithel bestehen und ein ausgereiftes fibröses Stroma aufweisen,

- die sowohl odontogenes Epithel als auch odontogenes Ektomesenchym als Tumorkomponenten besitzen und eine

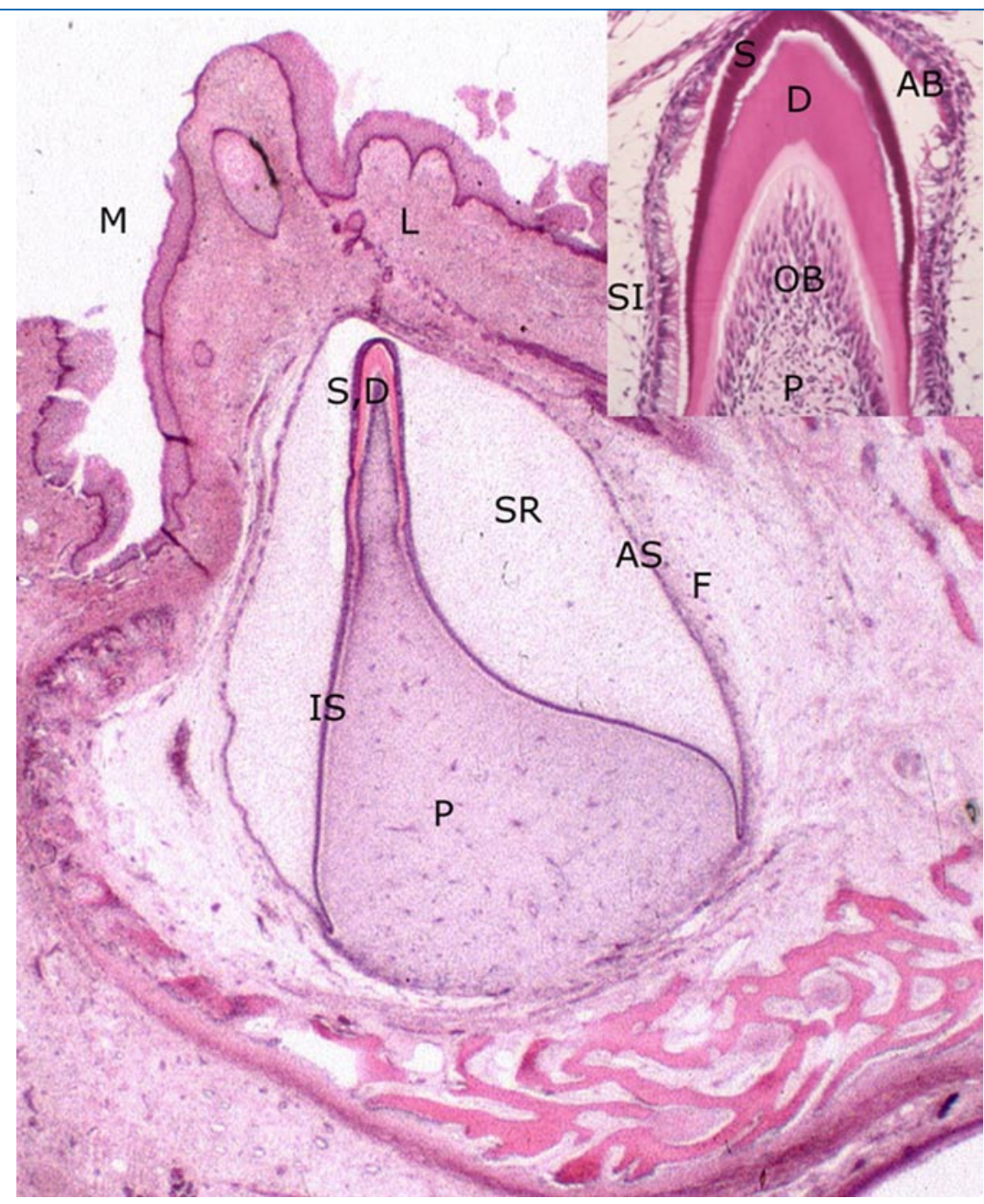

Abb. 1 \ Zahnentwicklung: Glockenstadium mit beginnender Bildung der Zahnkrone (M Mundschleimhaut, $L$ Zahnleiste, S,D Schmelz und Dentin, SR Stratum reticulare, $A S$ äußeres Schmelzepithel, F Zahnfollikel, IS inneres Schmelzepithel, P Pulpa/Ektomesenchym, $A B$ Ameloblasten, SI Stratum intermedium, $O B$ Odontoblasten)

Hartsubstanzbildung aufweisen können („gemischte" odontogene Tumoren) und solchen,

- die aus odontogenem Ektomesenchym bestehen bzw. rein mesenchymal sind und in die odontogenes Epithel als Hinweis auf ihren Ursprung eingeschlossen sein kann.

Epidemiologische Angaben über odontogene Tumoren liegen kaum vor. Die meisten Publikationen geben relative Häufigkeiten an, die aber durch lokale (z. B. Erreichbarkeit und Verfügbarkeit medizinischer Versorgung) und andere Besonderheiten (z. B. Spezialabteilungen) nur sehr schwer vergleichbar sind $[2,6]$. Aus diesem Grund zeigen die in der Literatur angege- benen Häufigkeiten für odontogene Tumoren eine sehr große Bandbreite [5].

Im Vergleich zur Klassifikation odontogener Tumoren von 1992 wurden in der neuen 3. Auflage eine Reihe von Umstellungen und Reklassifikationen vorgenommen, die zu Umgruppierungen, Dignitätsänderungen, Neuklassifizierungen, aber auch zu Vereinfachungen geführt haben.

In den nachfolgenden Artikeln werden die odontogenen Tumoren vor dem Hintergrund der neuen WHO-Klassifikation vorgestellt. Die ersten 3 Artikel behandeln die gutartigen odontogenen Tumoren, wobei den Prinzipien der Klassifikation folgend zunächst die rein epithelialen, dann die "gemischten" (epithelial und ektomesenchymal) und schließlich die mesenchymalen Tumoren diskutiert werden. Daran 
schließt sich die Darstellung der (noch selteneren) malignen odontogenen Tumoren an. Der Abschlussartikel hat dann die Zysten im Kiefer zum Thema.

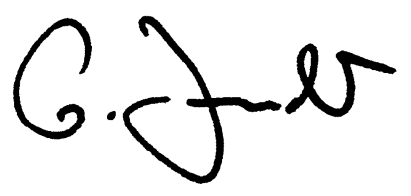

Gernot Jundt, Basel

\section{Korrespondenzadresse \\ Prof. Dr. G. Jundt}

Institut für Pathologie, DÖSAK-Zentralregister am Knochentumor-Referenzzentrum,

Universitätsspital Basel

Schoenbeinstraße 40, 4031 Basel, Schweiz

gernot.jundt@unibas.ch

\section{Literatur}

1. Barnes L, Eveson JW, Reichart P et al. (eds) (2005) Pathology and genetics of head and neck tumours. IARC, Lyon

2. Daley TD, Wysocki GP, Pringle GA (1994) Relative incidence of odontogenic tumors and oral and jaw cysts in a Canadian population. Oral Surg Oral Med Oral Pathol 77: 276-280

3. Kramer IRH, Pindborg JJ, Shear M (1992) Histological typing of odontogenic tumors. Springer, Berlin Heidelberg New York

4. Nanci A (2003) Ten cate's oral histology. Development, structure and function. Mosby, St. Louis

5. Reichart PA, Philipsen HP (2004) Odontogenic tumors and allied lesions. Quintessence, London Berlin Chicago Copenhagen

6. Speechley M, Stephens R (1995) What is „relative incidence"? Oral Surg Oral Med Oral Pathol Oral Radiol Endod 79: 2
(Hrsg.) B. Brinkmann, A. M. Raem,

R. Dettmeyer, H. Pfeiffer, U. Bux,

N. Westphal, G. Steinhilper

\section{Leichenschau}

Leitlinien zur Qualitätssicherung

Düsseldorf: Deutsche Krankenhaus Verlagsgesellschaft mbH 2007, 238 S., 75 Abb., (ISBN 978-3-935762-97-7), 45.00 EUR

Jede 2. Tötung bleibt in Deutschland unerkannt. Bundesweite einheitliche Regelungen zur Leichenschau gibt es immer noch nicht. Insofern sind die vorgelegten „Leitlinien zur Qualitätssicherung" sowohl medizinisch als auch gesamtgesellschaftlich wichtig, da sie eine zeitgemäße Leichenschau darstellen, mit der naturgemäß auch jeder Pathologe vertraut sein muss. Dem handlichen Buch sind nicht weniger als 5 (!) Geleitworte und ein Vorwort honoriger Juristen und Mediziner vorangestellt, die unisono die Notwendigkeit und Zweckmäßigkeit des standardisierten Leitlinienwerks preisen.

Kapitel I skizziert die rechtlichen Rahmenbedingungen bei natürlichen und bei vermuteten nichtnatürlichen Todesfällen unter Berücksichtigung von Gesetzestexten, praktischen Hinweisen zur Leichenschau, Todesarten und Obduktionsformen. Beispielhafter Leichenschaufehler ist das Übersehen hellroter Totenflecke bei Kohlenmonoxidintoxikation. Einschüsse werden als Platzwunden verkannt. Bei unterkühlten und/oder vergifteten Personen sind fälschlich Todesbescheinigungen ausgestellt worden, obwohl die Betroffenen noch lebten.

Kapitel II ist mit dem entsprechenden Abbildungsanhang das Kernstück des Buches. Es enthält die theoretischen und praktischen Leitlinien der Leichenschau von der Entkleidung der Leiche bis zur Darstellung der speziellen Fallgruppen nichtnatürlicher Todesfälle sowie plötzlicher Tode außerhalb und innerhalb des Krankenhauses. Für die äußere Besichtigung des entkleideten Leichnams wird eine sog. ZKH-TOUR propagiert. In diesem Akronym stehen $Z$ für Zeichen des Todes, K für Kopf, $\mathrm{H}$ für Hals - und TOUR für das weitere systematische Vorgehen bei der Leichenschau "Thorax, Obere, Untere Extremität und Rücken". Bauch und Körperöffnungen berücksichtigt die Eselsbrücke nicht, führt diese aber in der beigegebenen Tabelle mit möglichen Befunden auf. Alle auffälligen äußerlichen Leichenveränderungen mit Verdacht auf einen nichtnatürlichen Tod sind gesondert im Text markiert.

Kapitel III schildert die polizeiliche Todesermittlung, Kapitel IV bespricht gesondert die Qualitätssicherung der Leichenschau. Häufigkeitsangaben zu unerkannten Tötungen bewegen sich in einer Grauzone. Dass jede 2. Tötung in Deutschland unerkannt bleibe, stammt aus dem Geleitwort des früheren Generalbundesanwalts Kay Nehm. Hingegen dürfte nach Norbert Westphal, Kriminaldirektor in Münster, ,', das Dunkelfeld der vorsätzlichen Tötungsdelikte" bezogen auf absolute Zahlen eher geringfügig sein. Die offensichtliche Diskrepanz beider Meinungen ist nicht allein durch Definitionsfragen unterschiedlicher Tötungsarten zu verstehen. Mangelhafte Befunderhebung bei der Leichenschau, fehlende Anzeigenbereitschaft, Einflussnahme von Tätern und Wehrlosigkeit von Opfern seien Ursachen für Fehler beim Erkennen von Tötungshandlungen.

Allgemeinärzte führen durchschnittlich 9-mal im Jahr eine Leichenschau durch, die mittels der minutiösen Honorarkalkulationen des Kapitels V abzurechnen ist. Begrüßenswert erscheint ein angefügter bundesweit empfohlener Gesetzesentwurf zur ärztlichen Leichenschau. Bereichernd und hilfreich für die ordnungsgemäße Durchführung einer Leichenschau ist der Anhang mit instruktiven Farbbildern unterschiedlicher nichtnatürlicher Todesfälle. Die Abb. 72 zeigt das paradoxe forensische Unikat eines überlebten Zungensteckschusses: Nach einem Genickschuss-Mordversuch wurde der Einschuss am Hals chirurgisch als „Nackenplatzwunde" angesehen, während man das Projektil erst Tage später in der Muskulatur der linken Zungenseite entdeckte!

Leider fehlt dem praktischen Buch ein Stichwortverzeichnis für den Text- und Bildteil, das für eine Neuauflage unbedingt vorgesehen werden sollte. Zusammengefasst liegt eine zweckmäßige und informative leitlinienartige Darstellung zur Leichenschau vor, die bei entsprechender Nutzung eine Qualitätserhöhung dieser ärztlichen Leistung für alle Beteiligten ermöglicht.

H. Nizze (Rostock) 